\title{
Carboxymethyl guar gum nanoscaffold as matrix for cell growth in vitro
}

\begin{abstract}
Scaffolds are an integral part of tissue engineering, because they can mimic the functions of the extracellular matrix on which a cell grows. Scaffolds are used either as a matrix for the growth and proliferation of cells, or as a delivery system for growth factors to help cells grow. Polysaccharide scaffolds are practical, efficient and do not pose the problem of eliciting an immune response. Guar gum is a hydrated biopolymer obtained from the seeds of the Cyamopsis tetragonoloba, which is used in various fields. The guar gum can be modified easily to get a unique polymer which is structurally suitable for use in tissue engineering. The various structural characteristics of guar gum led us to attempt to design a scaffold using it. This scaffold was further functionalized with a carboxymethyl group, thus forming the carboxymethyl guar gum (CMGG) scaffold, with an average pore diameter of $10 \mu \mathrm{m}$. The aim of this paper is to assess the effects of the scaffold on the growth of RAW 264.7 macrophage cells, NIH3T3 fibroblast cells and bone marrow- derived mesenchymal stem cells. RAW 264.7 cells, NIH3T3 cells and BM-MSCs (untreated, treated with LPS to induce inflammation, and treated with $\mathrm{CoCl}_{2}$ to induce hypoxia) were grown on the scaffolds in 24 well plates, and observed for at least 48 hours. MTT assay was done to assess their ability to proliferate on the scaffolds. All the cell types were found to have successfully grown and proliferated on the scaffold, even under inflammatory and hypoxic conditions (induced by treating the cells with $1 \mu \mathrm{g} / \mathrm{ml}$ lipopolysaccharide and $100 \mathrm{mM}$ $\mathrm{CoCl}_{2} \cdot 6 \mathrm{H}_{2} \mathrm{O}$, respectively). These preliminary studies have showed that the carboxymethyl guar gum scaffold may be used as a matrix in tissue engineering.
\end{abstract}

Keywords: biomatrix, scaffold, biopolymers, hydrogels, cell migration, inflammation; cellular adherence and fibroblasts
Volume 5 Issue I - 2018

Mitra S, Ghosh N, Banerjee ER

Department of Zoology, University of Calcutta, India

Correspondence: Ena Ray Banerjee, Immunology \& Regenerative Medicine Research Laboratory, Department of Zoology, University of Calcutta, 35, Ballygunge Circular Road, Kolkata- 700019, West Bengal, India, Tel +9l-9163739975,

Email enaraybanerjee@gmail.com

Received: December 01, 2017 | Published: January 19, 2018
Abbreviations: CMGG, carboxymethyl guar gum; BM-Mscs, bone marrow derived mesenchymal stem cells; DMEM, dulbecco's modified eagle medium; FBS, foetal bovine serum; Pen-Strep, penicillin-streptomycin; LPS, Lipopolysaccharide; $\mathrm{Cocl}_{2} \cdot 6 \mathrm{H}_{2} \mathrm{O}$, cobalt chloride hexahydrate; PBS, phosphate buffered saline; EDTA, ethylene diamine tetra acetic acid; MTT, 3-(4, 5-Dimethylthiazol-2Y1)-2, 5-Diphenyltetrazolium Bromide; UV: ultraviolet light; Hcl: hydrochloric acid

\section{Introduction}

Polymeric scaffolds can be used in tissue engineering as a substitute to the extracellular matrix of the cell, as its porosity and large surface area can provide a support for the growth of the cells, and its surface properties and geometric structure direct the cells towards regeneration. ${ }^{1}$ Typically, scaffolds are designed as meshes, fibres or sponges, which allow the cells and nutrients to be, distributed uniformly. ${ }^{1}$ Polysaccharide hydrogels are very practicable tools for the design of scaffolds for tissue engineering, as they have macromolecular structures similar to native tissues..$^{2-4}$ In contrast to the protein matrices, most polysaccharide hydrogels are risk free and elicit low or no immunogenic reactions. Hydrogels can also be bioconjugated to improve their performance. ${ }^{4}$ Biopolymers like alginates, hyaluronic acid and cellulose are amply available in nature and can easily form porous biocompatible gel networks. ${ }^{5}$ Alginate biohybrids and covalently modified hyaluronates have been investigated earlier in tissue engineering arenas. Layered tissues can be formed using cell sheets. In this method, calcium alginate hydrogels can be used as a substrate for the growth of cells. ${ }^{6}$ Once the cells reach confluency, the hydrogel scaffold can be removed by using a chelating agent on the calcium, leaving the cells to proliferate and structure themselves into the tissue. ${ }^{6}$ Cellulose nano-formations and metal cross- linked hydrogels are other examples that are currently accepted for the design of engineered biocompatible materials.?

Tissue engineering is a multidisciplinary field that applies the principles of engineering and life sciences to develop biological substitutes that repair, maintain, or improve the function of a tissue or a whole organ. ${ }^{8}$ Organ failure and loss of tissue, caused due to an injury or any other type of damage, is a major life threatening human health problem. The solution to these problems is tissue or organ transplantation. Other therapies, like drug therapy or the expensive procedure of replacement by medical devices are not able to restore all the functions of a damaged or lost organ or tissue. Thus engineering cells, in combination with scaffolds, are currently used as a promising alternative in the field of tissue engineering, which can be used to treat the loss of a tissue or organ without the limitations of current therapies.

The field of regenerative medicine seeks to heal, replace, or regenerate tissues and organs damaged by injury or disease. Stem cells have been found to be a promising source of cells to address the challenges of tissue engineering. ${ }^{9}$ Stem cells can self-renew and differentiate into multiple lineages. This makes them an ideal source for cell therapy and regenerative medicine. ${ }^{10,11}$ Bone marrow derived mesenchymal stem cells (BM- MSCs) can be grown easily in cell culture dishes. By varying the composition of the medium in 
which they are grown, MSCs can be selectively differentiated into fat cells (adipocytes), bone cells (osteocytes), and cartilage cells (chondrocytes). ${ }^{12}$ Thus bone marrow is a good source of cells for skeletal tissue engineering. Stem cells are also known to be distributed around the body in various other 'niches'. However, stem cells cannot be grown easily in vitro and are thought to have a limited replicative capacity. ${ }^{12}$ The standard in vivo culture system involves growing cells in a monolayer on a charged, flat, plastic surface, although this cannot replicate the complexity of the cells' natural environment and rarely supports the transformation of the cells into a functional tissue. ${ }^{13}$ This can be achieved by providing an appropriate scaffold that will lead to the development of a functional tissue. This process is certainly not a simple matter, but this approach may overcome the problem, using a variety of materials. ${ }^{13}$

Scaffolds play an important role in tissue engineering with the goal of manipulating cell behaviour by creating a micro-environment to allow the cells to attach and subsequently develop into a tissue. ${ }^{14}$ There are mainly two approaches to using scaffolds in regenerative medicine. First, scaffolding can be used as a structural support upon which cells are seeded in vitro. Here, the matrix provides the foundations tissue development. Secondly, using the scaffold as a delivery device for growth factors. This strategy involves combining the scaffold with growth factors, so that, upon implantation, cells from the body are recruited to the scaffold site to form a tissue throughout the matrix. ${ }^{14}$ Scaffolds are typically made up of polymeric bio-materials such as poly lactic-co-glycolic acid (PLGA) and poly lactic acid (PLA). Scaffolds may also be of other types, like high-pressure $\mathrm{CO}_{2}$ foamed scaffolds, injectable scaffolds, and novel custom scaffolds. These have been extensively used for tissue engineering. ${ }^{14}$

Nanoscaffolds have been used in the field of tissue engineering and regenerative medicine for quite some time, with satisfactory results. Nanoscaffolds can provide a suitable microenvironment for the growth and proliferation of cells. Several types of scaffolds, including biodegradable ones, have been made. Nanoscaffolds have to be designed in such a way that they have small pore sizes, so the cells cannot escape into the empty spaces, and so that they have no immunological side-effects. Many scaffolds have been used in mesenchymal stem cell- based therapy, where MSCs have been cultured on scaffolds and differentiated into different cell types. ${ }^{15}$

Tissue engineering has long been used in healing of skin wounds. Scaffolds can be used to mimic the skin cells, and provide a matrix for the tissue to grow. These scaffolds can be produced from natural materials, like collagen and gelatin, or syntheti/polymeric materials like polyglycolic acid, polylactic acid, hydrogels or selfassembling peptides. These materials generally do not pose the problem of rejection, and are biocompatible. ${ }^{16}$ Guar gum $(\mathrm{GG})$ is a hydrated biopolymer obtained from the seeds of the bean Cyamopsis tetragonoloba, composed of a straight chain of $\beta-1-4$-mannose with a single galactose unit linked to alternate mannose units by $\alpha-1-6$ linkages. ${ }^{17}$ The biopolymer is used regularly as functional food, ${ }^{17}$ as well as in cosmetics ${ }^{17}$ and drug delivery. ${ }^{17-19}$ Inter- and intra- molecular hydrogen bonding of the galactose units of GG allow for its hydration in a three chain coil formation. Despite being a non- ionic polymer, GG interacts and binds with proteins and lectins in a physiological environment due to its glycopolysaccharide structure. ${ }^{20,21}$ This ability of GG to interact with proteins has been used to design artificial tear to treat dry eyes. ${ }^{22}$

With these unique structural characteristics in mind, we used GG to design a scaffold to be used for tissue engineering. The GG was modified in such a way as to confer a high degree of functionality to it, to allow for better interaction with molecules. The scaffold used in this study was functionalised with carboxymethyl group, and thus, named carboxymethyl guar gum (CMGG) nanoscaffold. This form of the scaffold has an average pore diameter of $10 \mu \mathrm{m}$. The present study aims to assess how cell-matrix interactions influence the in vitro behavior of different cell types (RAW 264.7 macrophage cell line, NIH3T3 fibroblast cell line and BM-MSCs) grown on CMGG nanoscaffold, and how they react under the effects of inflammation and hypoxia.

\section{Materials and methods}

\section{Materials}

Dulbecco's Modified Eagle Medium (DMEM), foetal bovine serum (FBS), and Penicillin- Streptomycin (Pen- Strep) were bought from Himedia, India. Bacterial lipopolysaccharide (LPS) was obtained from Sigma Aldrich; cobalt (II) chloride hexahydrate $\left(\mathrm{CoCl}_{2} .6 \mathrm{H}_{2} \mathrm{O}\right)$ was purchased from Merck, India. Phosphate buffered saline (PBS; $\mathrm{pH}$ 7.4) was purchased from Himedia (India). 24 well plates were obtained from Nest Biotech Co. Ltd., China, and were incubated in a $\mathrm{CO}_{2}$ incubator (Thermo Fisher) at $37^{\circ} \mathrm{C}$, with $5 \% \mathrm{CO}_{2}$. Cells scrapers from Himedia, India were used to detach cells. All cell-culture work was done inside the biosafety cabinet. CMGG scaffold was a kind gift from Dr. Arup Mukherjee, Calcutta University (India).

Cell Lines: RAW 264.7 macrophage cells were cultured in our laboratory. NIH3T3 cells were obtained from NCCS, Pune (India) and cultured in our laboratory. Bone marrow mesenchymal stem cells (BM-MSCs) were isolated from adult male BALB/c mice, purchased from National Institute of Nutrition (Hyderabad, India) and cultured in our laboratory.

\section{Methods}

Ethical statement for animal experiments: All experiments using mice (for isolation of BM- MSCs) were performed according to rules laid down by the animal ethics committee of the Department of Zoology, University of Calcutta, and the animals housed under specific pathogen free conditions at the animal house of the Department of Zoology, University of Calcutta.

Culture of cells: RAW 264.7 macrophage cells were cultured in DMEM, with $10 \%$ FBS and $1 \%$ Pen- strep, and incubated at $37^{\circ} \mathrm{C}$ with $5 \% \mathrm{CO}_{2}$, till confluent. On reaching the confluence, the cells were scraped out, washed with $1 \mathrm{X}$ PBS and seeded in the wells. NIH3T3 cells were also cultured in complete DMEM (DMEM + $10 \%$ FBS $+1 \%$ Pen-Strep) and incubated at $37^{\circ} \mathrm{C}$ with $5 \% \mathrm{CO}_{2}$, till confluent. On reaching the confluence, the cells were trypsinized using $1 \mathrm{X}$ Trypsin EDTA solution $(0.25 \%$ Trypsin and $0.2 \%$ EDTA in Dulbecco's Buffered saline), washed with DMEM complete medium and seeded in respective wells. Bone marrow mesenchymal stem cells (BM- MSCs) were derived from BALB/c mice by gently flushing out the bone marrow with DMEM. The bone marrow cells were then cultured in complete DMEM (DMEM $+15 \%$ FBS $+1 \%$ Pen-Strep) with periodic media change to eliminate the hematopoietic cells. From day 3, spindle shaped MSCs appeared which was and incubated at $37^{\circ} \mathrm{C}$ with $5 \% \mathrm{CO}_{2}$, till $70 \%$ confluent. On reaching confluence, the cells were trypsinized, washed and used for assays.

Culturing RAW 264.7 cells on scaffolds for 3 days: The CMGG 
scaffolds were UV sterilized for $15 \mathrm{~min}$ in the biosafety cabinet, cut into small pieces and plated in a 24 well plate which was labeled for day 1 , day 2 and day 3 . The pieces were further washed with 1 X PBS and then incubated overnight in DMEM, at $37^{\circ} \mathrm{C}$ in a humidified $5 \%$ $\mathrm{CO}_{2}$ incubator prior to cell seeding. This was done to facilitate cell attachment onto the nanofibers. Next day, $5 \times 10^{4}$ RAW 264.7 cells were seeded in the well labeled for dayl, same number of cells in the well with scaffold as well as the control well which was without scaffolds for day 1 . The seeding density was properly maintained for wells labeled for rest of the days and the culture medium was changed regularly. The cells were observed at 20X of an inverted microscope (Nikon Eclipse TiS) and photographed with Nikon DS Fi2 camera for cell quantification.

Culturing NIH3T3 cells on scaffolds for 3 days: The CMGG scaffolds were UV sterilized for $15 \mathrm{~min}$ in the biosafety cabinet, cut into small pieces and plated in a 24 well plate which was labeled for day 1,2 , and day 3 . The pieces were further washed with $1 \mathrm{X}$ PBS and then incubated overnight in DMEM, at $37^{\circ} \mathrm{C}$ in a humidified $5 \%$ $\mathrm{CO}_{2}$ incubator prior to cell seeding. This was done to facilitate cell attachment onto the nanofibers. Next day, the $5 \times 10^{4}$ NIH3T3 cells were seeded in the well labeled for day1, same number of cells in the well with scaffold as well as the control well which was without scaffolds for day 1 . The seeding density was properly maintained for wells labeled for rest of the days and the culture medium was changed regularly. The cells were observed at 20X of an inverted microscope (Nikon Eclipse Ti S) and photographed with Nikon DS Fi2 camera for cell quantification.

Culturing Bone marrow derived mesenchymal stem cells (BMMSCs) on scaffolds for 3 days: The mesenchymal stem cells were obtained by harvesting the bone marrow from Balb/c mice. The whole bone marrow cells were cultured in DMEM medium supplemented with $15 \%$ FBS and $1 \%$ Pen-Strep. The media was changed and refeeding of cells with fresh medium was followed for 3 days to exclude the haematopoetic cells. The MSCs shows plastic adherent property which helps them to remain adhered to the plate and proliferate. Microscopic visualization was done periodically for morphological examination. The cells were further maintained in the above mentioned medium and used for MTT assay as well as treated with different stress factors.

MTT Assay for RAW 264.7 cells, NIH3T3 cells and BM-MSCs grown on scaffold for 3 days: Seeding of cells were done as mentioned above and the respective wells were allowed to grow for 24 hours in $\mathrm{CO}_{2}$ incubator. They were imaged microscopically to examine the colonization of cells with and without scaffold. The cells were treated with $40 \mu 1$ of $0.5 \mathrm{mg} / \mathrm{ml}$ MTT reagent (in 1 X PBS) and incubated for 3 hours at $37^{\circ} \mathrm{C}, 5 \% \mathrm{CO}_{2}$. The media was discarded and the formazan dye was dissolved with acidic Isopropanol $(0.04 \mathrm{M}$ $\mathrm{HCl}$ in Isopropanol). Then $100 \mu 1$ of dissolved dye was transferred in triplicates to fresh 96 well plate and the absorbance was measured at $540 \mathrm{~nm}$ in Microplate Reader (Thermo-Fisher Scientific). The assay was done for all the 3 days.

Treatment of bone marrow derived MSCs: To assess the effects on inflammation and hypoxia on the growth of the cells in scaffold, and to determine whether the scaffold plays any protective role in stress conditions, such stress conditions were induced. The cells were divided into the following treatment groups:
A. Only BM- MSCs
B. BM- MSCs + CMGG scaffold

\section{BM- MSCs + CMGG scaffold $+1 \mu \mathrm{g} / \mathrm{ml}$ LPS \\ D. BM- MSCs + CMGG scaffold $+100 \mu \mathrm{M} \mathrm{CoCl}_{2} \cdot 6 \mathrm{H}_{2} \mathrm{O}$}

\section{E. Only CMGG Scaffold}

For the CMGG groups, the wells were incubated overnight with the CMGG scaffold. $0.1 \times 10^{6}$ cells/ $\mathrm{ml}$ of the isolated BM- MSCs were seeded in the respective wells of the 24- well plates. The cells were treated with $1 \mu \mathrm{g} / \mathrm{ml}$ lipopolysaccharide for $18 \mathrm{hrs}$ to induce inflammation. ${ }^{23,24}$ The cells were treated with $100 \mu \mathrm{M}$ cobalt chloride for $24 \mathrm{hrs}$ to induce hypoxia. ${ }^{25-27}$ The plates were then placed in a $\mathrm{CO}_{2}$ incubator at $37^{\circ} \mathrm{C}$ with $5 \% \mathrm{CO}_{2}$, and the cells were observed for over a period of 24 hours and 48 hours. The cells were observed at $20 \mathrm{X}$ of an inverted microscope (Nikon Eclipse Ti-S) and photographed with Nikon DS-Fi2 camera for cell quantification.

Statistical analysis: All data have been presented as Mean \pm Standard error of mean (SEM). Statistical significance has been calculated using t-test, considering all values of $\mathrm{p}<0.05$ to be significant.

\section{Results}

\section{Proliferation and viability of RAW 264.7 cells cultured on the CMGG sacaffold}

RAW 264.7 cells are the murine macrophage cells which are easy to propagate for inflammatory assay. We have cultured these cells both in absence and presence of scaffolds. The aim is to get an idea if the CMGG scaffolds are suitable for the growth of macrophages. The study was done for $72 \mathrm{hrs}$ where it was found that the scaffold is providing matrix for adherence and growth of cells on them. The morphology of the cells was monitored microscopically where the cells showed proliferation on the scaffold as compared to the control cells without scaffold (Figure 1). MTT assay was done to quantify the cellular viability of RAW 264.7 cells with and without scaffold as well as to check if the CMGG scaffolds shows any cytotoxic effect on RAW 264.7 cells. There was a 4.18 fold increase $(p<0.05)$ in the cell proliferation of RAW cells cultured on scaffold for $24 \mathrm{hrs}$ as compared to the cells without scaffold. After $48 \mathrm{hrs}$ and $72 \mathrm{hrs}$, there was a 1.58 fold increase in the cell proliferation and the fold change was significant for $72 \mathrm{hrs}$ (Figure 2 \& Table 1). Thus it can be said that the CMGG scaffold is not affecting the viability of the cells and promoting growth and proliferation of RAW 264.7 cells in vitro.

\section{Proliferation and viability of NIH3T3 cells cultured on the CMGG scaffold}

Fibroblast cells are mainly concerned for tissue metabolism and the structural integrity of the tissue, by secreting precursors for extra cellular matrix. In this study, we have used a NIH3T3 cell line, which is a mouse embryonic fibroblast cell to check the effect of CMGG scaffolds on the growth and proliferation of fibroblasts. On day 0, $0.5 \times 10^{5}$ cells were seeded on scaffold as well as without scaffold. The cells were cultured for $72 \mathrm{hrs}$, and the growth and proliferation was monitored microscopically after $24 \mathrm{hrs}, 48 \mathrm{hrs}$ and $72 \mathrm{hrs}$ (Figure 3). The MTT assay was done to quantify the viability of cells at the given interval of time. There was significant increase in the proliferation of cells when cultured on CMGG scaffold as compared to without scaffold. On day 1 , there was a 1.5 fold increase $(p<0.05)$ in the viability of NIH3T3 cells which was increased to 2.8 fold $(p<0.05)$ on day 2 as compared to cells culture without scaffold. On day 3 , there was a 2.8 fold increase $(\mathrm{p}<0.05)$ as compared to the cells cultured without scaffold (Figure $4 \&$ Table 2). Thus it can be said that CMGG 
scaffold is not affecting the viability of cells and is providing a suitable matrix for the fibroblast and may be used for tissue engineering.

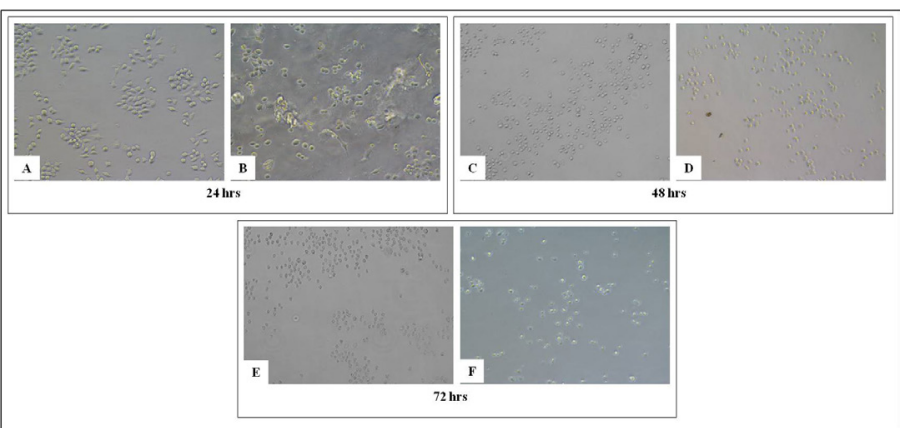

Figure I Growth of RAW 264.7 cells on tissue culture treated plate (A, C and E) without CMGG Scaffolds, (B, D and F) with CMGG Scaffolds on Day I, 2 and 3. Microscopic images captured at 20X of Nikon Eclipse TiS inverted microscope.

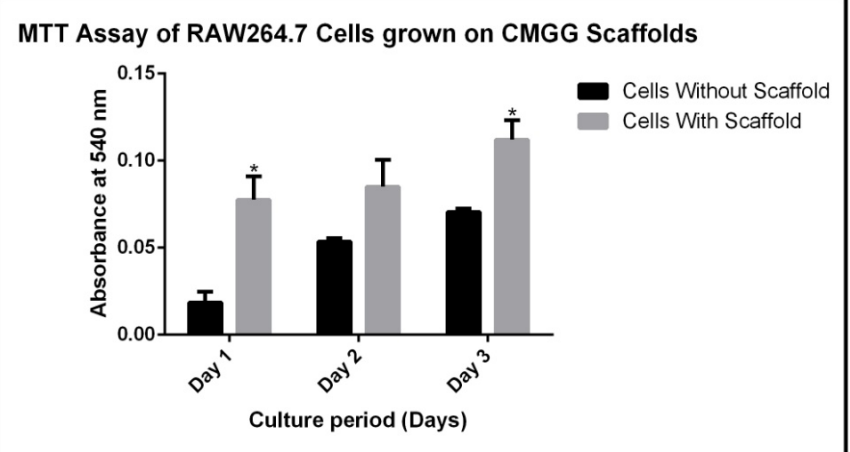

Figure 2 MTT assay of RAW 264.7 cells cultured on CMGG scaffold. The error bars represent the Mean \pm SEM $(n=3$; *denotes $p<0.05$ with respect to cells without scaffold).

Table I Fold change in the MTT assay of RAW 264.7 cells culture on scaffold with respect to cells cultured without scaffold. RAW cells cultured on scaffold shows significant increase in proliferation after $24 \mathrm{hrs}$ and $72 \mathrm{hrs}(* \mathrm{*}<0.05$ with respect to cells without scaffold)

\begin{tabular}{llll}
\hline & \multicolumn{2}{l}{ Absorbance at 540nm } & \multicolumn{2}{l}{$\begin{array}{l}\text { Fold change with } \\
\text { respect to cells } \\
\text { without scaffold }\end{array}$} \\
\cline { 2 - 4 } & Without scaffold & With scaffold & $(+) 4.18^{*}$ \\
\hline Day 1 & $0.019 \pm 0.005$ & $0.078 \pm 0.009$ & $(+)$ \\
Day 2 & $0.054 \pm 0.001$ & $0.085 \pm 0.011$ & $(+) 1.58$ \\
Day 3 & $0.071 \pm 0.001$ & $0.112 \pm 0.008$ & $(+) 1.58^{*}$ \\
\hline
\end{tabular}

MTT: 3-(4, 5-dimethylthiazol-2-yl)-2, 5-diphenyltetrazolium Bromide

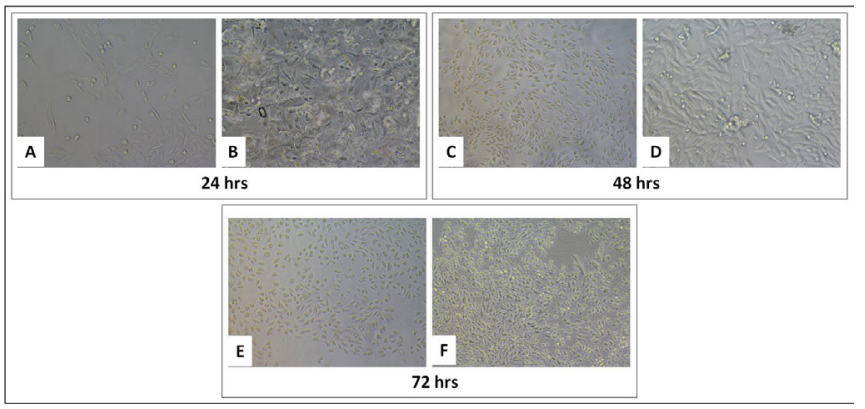

Figure 3 Growth of NIH3T3 cells on tissue culture treated plate (A, C and E) without CMGG Scaffolds, (B, D and F) with CMGG Scaffolds on Day I, 2 and 3. Microscopic images captured at 20X of Nikon Eclipse TiS inverted microscope.

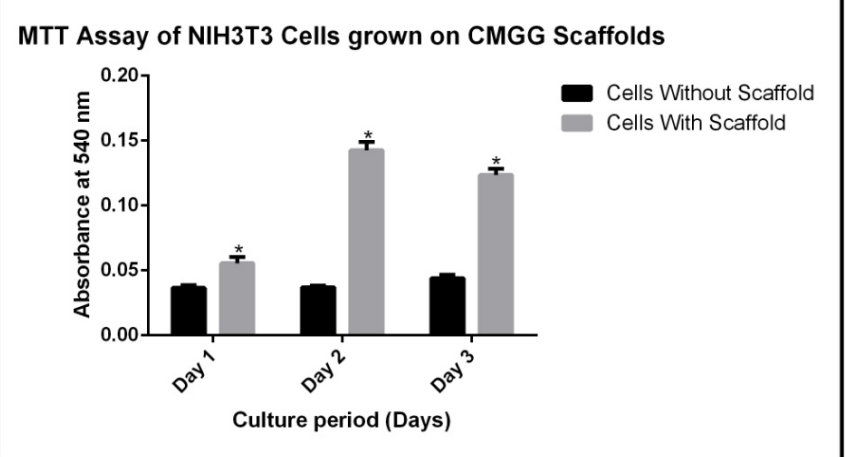

Figure 4 MTT assay of NIH3T3 cells cultured on CMGG scaffold. The error bars represent the Mean \pm SEM $(n=3$; *denotes $p<0.05$ with respect to cells without scaffold).

Table 2 Fold change in the MTT assay of NIH3T3 cells culture on scaffold with respect to cells cultured without scaffold. NIH3T3 cells cultured on scaffold shows significant increase in proliferation after $24 \mathrm{hrs}, 48 \mathrm{hrs}$ and 72 hrs (*: $\mathrm{p}<0.05$ with respect to cells without scaffold)

\begin{tabular}{|c|c|c|c|}
\hline & \multicolumn{2}{|c|}{ Absorbance at $540 \mathrm{~nm}$} & \multirow{2}{*}{$\begin{array}{l}\text { Fold change with } \\
\text { respect to cells } \\
\text { without scaffold }\end{array}$} \\
\hline & Without scaffold & With scaffold & \\
\hline Day 1 & $0.036 \pm 0.001$ & $0.056 \pm 0.004$ & $(+) 1.5^{*}$ \\
\hline Day 2 & $0.037 \pm 0.001$ & $0.143 \pm 0.005$ & $(+) 3.8^{*}$ \\
\hline Day 3 & $0.044 \pm 0.002$ & $0.124 \pm 0.004$ & $(+) 2.8^{*}$ \\
\hline
\end{tabular}

MTT: 3-(4, 5-dimethylthiazol-2-yl)-2, 5-diphenyltetrazolium Bromide

\section{Proliferation and viability of BM-MSCs cultured on the CMGG scaffold}

The bone marrow is a huge source of haematopoietic stem cells and mesenchymal stem cells. The cells are used mostly for tissue engineering studies for their self renewal property. The MSCs used in this study are derived from Balb/c mice and the MTT assay is done to check the effect of CMGG scaffold on growth and proliferation of BM-MSCs. The cells were cultured on CMGG scaffold for $72 \mathrm{hrs}$ with a periodic microscopic examination every $24 \mathrm{hrs}$ (Figure 5). The MTT assay was done to quantify the viability of cells at the given interval of time. There was a 2.6 fold increase in cell proliferation $(p<0.05)$ of cells when grown for $24 \mathrm{hrs}$ and there was 1.1 fold decrease in the cell proliferation when grown for $48 \mathrm{hrs}$, though the decrease was not significant (Figure $6 \&$ Table 3).

\section{Effect of LPS and CoCl2 on BMMSCs grown on CMGG scaffold}

The BM-MSCs are a promising area for tissue engineering studies for regenerative therapies. In this study, BM-MSCs are derived from $\mathrm{Balb} / \mathrm{c}$ mice and are cultured on CMGG scaffolds to check their proliferation to validate scaffolds in vivo for the revival of degenerated tissues through tissue engineering studies in tissue degenerative disorders. The two treatment groups are LPS $(1 \mu \mathrm{g} / \mathrm{ml})$ for $18 \mathrm{hrs}$ and $\mathrm{CoCl}_{2} \cdot 6 \mathrm{H}_{2} \mathrm{O}$ for $24 \mathrm{hrs}$ to induce inflammation and hypoxic condition respectively. The microscopic images are captured under $20 \mathrm{X}$ after the incubation period of cells with treatment groups (Figure 7). This was done to understand the ability of the scaffold to protect the cellular functions under stressed condition which are mainly found during tissue degeneration or injury. There was a 1.37 fold decrease in the cell proliferation as compared to cells grown without scaffold which was relevant with the time kinetics growth of BM-MSCs after 
$48 \mathrm{hrs}$ (Figure 5) where the proliferation was decreased after $48 \mathrm{hrs}$ of culture. In presence of LPS, there was a 1.86 fold increase in the cell proliferation and 2.36 fold $(\mathrm{p}<0.05)$ increase under hypoxia; the fold change was compared with cells grown without scaffold (Figure 8 \& Table 4).

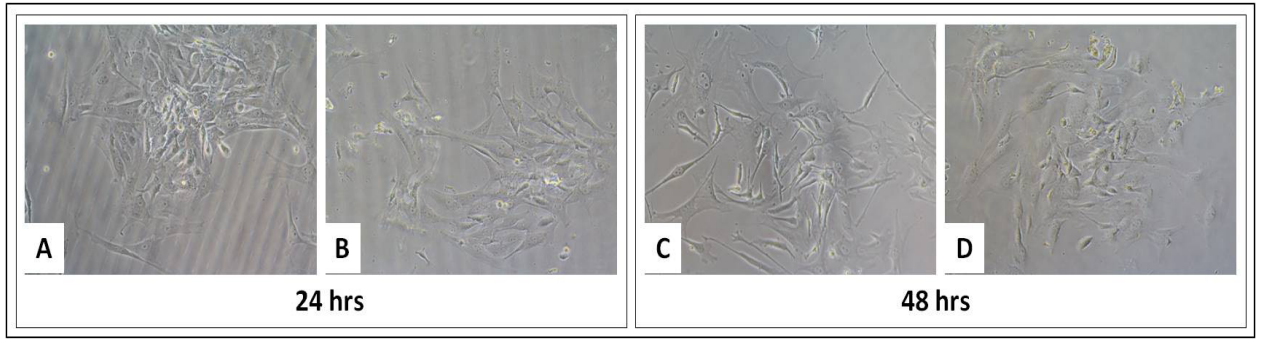

Figure 5 Growth of BM-MSCs on tissue culture treated plate (A and C) without CMGG Scaffolds, (B and D) with CMGG Scaffolds on Day I and 2. Microscopic images captured at 20X of Nikon Eclipse TiS inverted microscope.

\section{MTT Assay of BMMSCs grown on CMGG Scaffold}

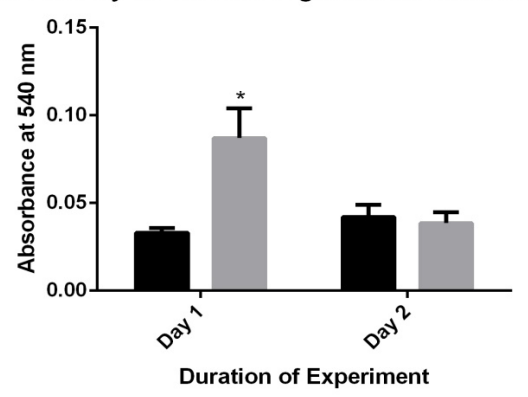

Figure 6 MTT assay of BM-MSCs cultured on CMGG scaffold. The error bars represent the Mean \pm SEM $(n=3$; * denotes $p<0.05$ with respect to MSCs without scaffold).

Table 3 Fold change in the MTT assay of BM-MSCs culture on scaffold with respect to cells cultured without scaffold. BM-MSCs cultured on scaffold shows significant increase in proliferation after $24 \mathrm{hrs}(*: \mathrm{p}<0.05$ with respect to cells without scaffold).

\begin{tabular}{llll}
\hline & \multicolumn{2}{l}{ Absorbance at 540nm } & \multicolumn{2}{l}{$\begin{array}{l}\text { Fold change with } \\
\text { respect to cells } \\
\text { without scaffold }\end{array}$} \\
\cline { 2 - 4 } & Without scaffold & With scaffold & $(+) 2.6^{*}$ \\
\hline Day I & $0.033 \pm 0.002$ & $0.087 \pm 0.012$ & $(-) 1.1$ \\
Day 2 & $0.042 \pm 0.042$ & $0.038 \pm 0.004$ & $\left(\begin{array}{l}\text {. } \\
\hline\end{array}\right.$
\end{tabular}

MTT: 3-(4, 5-dimethylthiazol-2-yl)-2, 5-diphenyltetrazolium Bromide; BMMSCs: Bone Marrow Derived Mesenchymal Stem Cells.

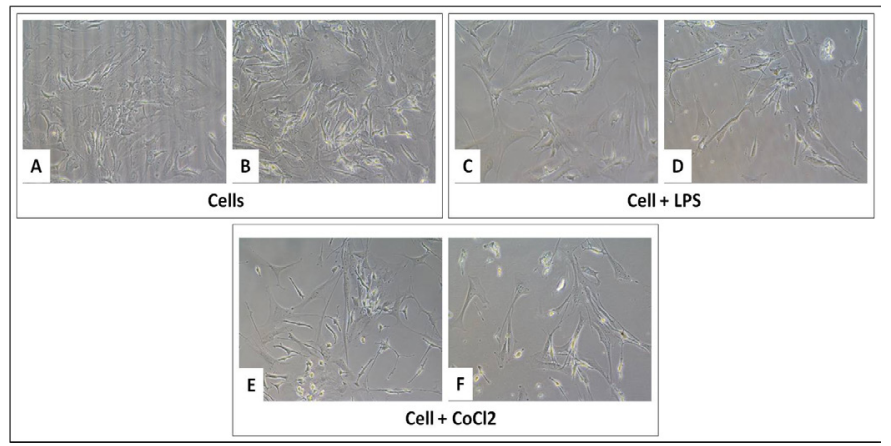

Figure 7 Growth of BM-MSCs on tissue culture treated plate without CMGG Scaffolds (A, C and E); with CMGG Scaffolds (B, D and F) after LPS treatment for $18 \mathrm{hrs}$ and $\mathrm{CoCl}_{2}$ for $24 \mathrm{hrs}$. Microscopic images captured at 20X of Nikon Eclipse TiS inverted microscope.
Effect of LPS and $\mathrm{CoCl}_{2}$ on MSCs grown on CMGG Scaffold

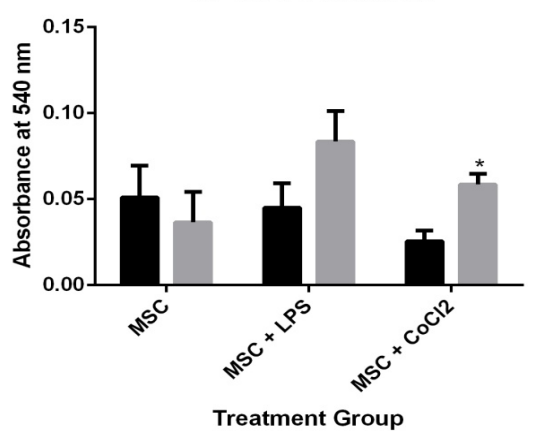

Figure 8 MTT assay of BMMSCs grown on CMGG scaffold under treatment of LPS for 18 hrs and $\mathrm{CoCl}_{2}$ for 24 hrs (* indicate $p<0.05$ with respect to MSCs without scaffold).

Table 4 Fold change in the MTT assay of BM-MSCs culture on scaffold under LPS and $\mathrm{CoCl}_{2}$ treatment with respect to cells cultured without scaffold. BM-MSCs cultured on scaffold showed significant increase in the fold change under hypoxic condition (*: $p<0.05$ with respect to cells without scaffold).

\begin{tabular}{|c|c|c|c|}
\hline \multirow[b]{2}{*}{ Treatment } & \multicolumn{2}{|c|}{ Absorbance at $540 \mathrm{~nm}$} & \multirow{2}{*}{$\begin{array}{l}\text { Fold change with } \\
\text { respect to cells } \\
\text { without scaffold }\end{array}$} \\
\hline & $\begin{array}{l}\text { Without } \\
\text { scaffold }\end{array}$ & $\begin{array}{l}\text { With } \\
\text { scaffold }\end{array}$ & \\
\hline MSC & $0.051 \pm 0.013$ & $0.037 \pm 0.013$ & (-) 1.37 \\
\hline MSC + LPS & $0.045 \pm 0.01$ & $0.084 \pm 0.013$ & $(+) 1.86$ \\
\hline $\mathrm{MSC}+\mathrm{CoCl} 2$ & $0.025 \pm 0.004$ & $0.059 \pm 0.005$ & $(+) 2.36^{*}$ \\
\hline
\end{tabular}

MTT:3-(4,5-dimethylthiazol-2-yl)-2,5-diphenyltetrazolium Bromide;BM-MSCs: Bone Marrow Derived Mesenchymal Stem Cells; LPS: Lipopolysaccharide; $\mathrm{CoCl}_{2}$ : Cobalt Chloride.

\section{Discussion}

In this study we have performed in vitro experiments to check the efficiency of CMGG scaffold as a bio-matrix to support the growth and adherence of cells on it. We have checked the cytocompatibility of scaffolds with RAW 264.7 macrophages, NIH3T3 fibroblast and BM-MSCs in vitro in this study. As the CMGG scaffolds are mannose rich, we tried to check the growth and proliferation of macrophages which expresses mannose receptor on them. The RAW 264.7 cells are the accepted model for inflammatory and cytotoxicity studies of the scaffold. ${ }^{28}$ In the first part of our experiment, we have performed microscopic studies for RAW 264.7 macrophage cells, followed by 
MTT assay to quantify the proliferation of cells on scaffold. The microscopic studies showed that they have adhered and proliferated on the scaffolds and the MTT assay showed significant increase in the fold change when cultured for $72 \mathrm{hrs}$. The NIH3T3 cells are the most suitable cells for the migratory, wound healing and proliferation studies. ${ }^{29}$ The fibroblasts have a tendency to attain a 3D morphology in a living system to communicate with their neighboring cells which is difficult when grown on a plate in $2 \mathrm{D} .^{30}$ The CMGG scaffolds provide that $3 \mathrm{D}$ biomatrix to ensure the colonization in vitro. We have checked the adherence and proliferation of NIH3T3 cells on CMGG scaffolds and a significant increase in the proliferation was observed when monitored for $24 \mathrm{hrs}$ to $72 \mathrm{hrs}$. Also the morphology of the cells microscopically showed that they were compatible with the CMGG scaffold which is supporting their adherence and proliferation. The bone marrow MSCs are used for differentiation towards different lineages when supplemented with the established lineage specific factors, thus BM-MSCs are considered as the potent cells for tissue engineering studies. ${ }^{31}$ We performed an experiment with BM-MSCs, to check if CMGG scaffolds are supporting the growth of BM-MSCs The BM-MSCs adhered on the scaffold and their proliferation is also increased significantly after $24 \mathrm{hrs}$ as compared to cells grown without scaffold. Studies showed that during inflammatory state, there is metabolic imbalance at the mucosal surface of the tissues causing hypoxia characterized by over expression of hypoxia inducible factor (HIF). ${ }^{32,33}$ The hypoxia preconditioned BM-MSCs have been used for tissue injury in previous studies considering the MSCs which survives the hypoxic stressed condition are adapted to counteract and survive under the conditions prevailing during the injury for its recovery. ${ }^{34}$ In our experiment we have checked the effect of hypoxia on cells as well as on scaffolds which was done after treating the cells grown on scaffold with $\mathrm{CoCl}_{2}$. We have also checked the effect of LPS which is a widely accepted inducing agent for inflammation. ${ }^{35}$ There was 1.86 fold increase in proliferation of BM-MSCs when treated with LPS and 2.36 fold increase in the fold change when treated with $\mathrm{CoCl}_{2}$, as compared to the untreated cells. Thus in vitro studies showed that the pore size of scaffold is suitable for cell adherence for RAW 264.7, NIH3T3 cells as well as BM-MSCs. Our study also showed that the scaffolds are least affected by the inflammatory as well as the hypoxic stimulus and serve as a suitable biomatrix for the maintenance of cellular morphology. Thus, from our results we can say that CMGG scaffold can sustain the stress conditions prevailing during the tissue injury and degenerative processes and may be used as a tool for tissue regeneration studies.

\section{Conclusion}

The emerging field of tissue engineering has gained interest to understand the possible combination of CMGG nanoscaffolds with cells. In this study, we have performed in vitro experiments to check the adherence of cells on the scaffold as well as their growth and proliferation on the scaffold matrix. The first stage of our experiment showed increased proliferation of two different types of murine cells- the RAW 264.7 macrophage cells and the NIH3T3 fibroblast cells, when cultured on CMGG scaffold for $72 \mathrm{hrs}$. Since scaffolds are mainly used in tissue engineering, where the main component is often stem cells, we further checked the proliferation of BM-MSCs derived from Balb/c mice on scaffold which showed increased proliferation when cultured for $24 \mathrm{hrs}$ and $48 \mathrm{hrs}$. From our studies we can conclude that, up to a certain time point, the CMGG scaffold is providing a functional $3 \mathrm{D}$ matrix supporting the adhesion, growth and proliferation of different types of cells. Thus our in vitro study confirms the cytocompatibility of CMGG scaffolds as a biomaterial. However in vivo study with CMGG scaffold in mice model will confirm whether it can be used for development of tissue engineering and regenerative medicines.

\section{Acknowledgement}

We wish to acknowledge Professor Arup Mukherjee, Department of Chemical Technology, University of Calcutta, and his research fellow Ms. Sonia Kundu, for supplying us with the CMGG nanoscaffold. We also wish to acknowledge University Grants Commission (UCG), New Delhi, for providing fellowship and contingency grant to SM, and Indian Council for Medical Research (ICMR), New Delhi, for providing a fellowship to NG.

\section{Conflict of interest}

The authors have declared that no conflict of interest exists.

\section{Contribution of authors}

SM \& NG performed the experiments, analysed the data and wrote the manuscript. ERB initiated the project with her idea, designed the experiments, analyzed all data and wrote the manuscript.

\section{References}

1. Dhandayuthapani B, Yoshida Y, Maekawa T, et al. Polymeric Scaffolds in Tissue Engineering Application: A Review. International Journal of Polymer Science. 2011(2011):1-19.

2. Balakrishnan B, Banerjee R. Biopolymer-based hydrogels for cartilage tissue engineering. Chem Rev. 2011;111(8):4453-4474.

3. Hoch E, Tovar GEM, Borchers K. Biopolymer-based hydrogels for cartilage tissue engineering. Bioinspired, Biomimetic and Nanobiomaterials. 2016;5(2):51-66.

4. Ahadian S, Sadeghian RB, Salehi S, et al. Bioconjugated hydrogels for tissue engineering and regenerative medicine. Bioconjugate Chem. 2015;26(10):1984-2001.

5. Foss C, Merzari E, Migliaresi C, et al. Silk fibroin/hyaluronic acid $3 \mathrm{~d}$ matrices for cartilage tissue engineering. Biomacromolecules. 2013;14(1):38-47.

6. Yan J, Chen F, Amsden BG. Cell sheets prepared via gel-sol transition of calcium RGD-alginate. Acta Biomaterialia. 2016;30:277-284.

7. Domingues RMA, Gomes ME, Reis RL. The potential of cellulose nanocrystals in tissue engineering strategies. Biomacromolecules. 2014;15(7):2327-2346.

8. Langer R, Vacanti JP. Tissue engineering. Science. 1993;260(5110):920-926.

9. Sundelacruz S, Kaplan DL. Stem cell and scaffold-based tissue engineering approaches to osteochondral regenerative medicine. Semin Cell Dev Biol. 2009;20(6):646-655.

10. Ali H, Al-Yatama MK, Abu-Farha M, et al. Multi-lineage differentiation of human umbilical cord wharton's jelly mesenchymal stromal cells mediates changes in the expression profile of stemness markers. PLoS One. 2015;10(4): $\mathrm{e} 0122465$.

11. Ullah I, Subbarao RB, Rho GJ. Human mesenchymal stem cells-current trends and future prospective. Biosci Rep. 2015;35(2):e00191.

12. Guillot PV, Cui W, Fisk NM, et al. Stem cell differentiation and expansion for clinical applications of tissue engineering. J Cell Mol Med. 2007;11(5):935-944.

13. Evans ND, Gentleman E, Polak JM. Scaffolds for stem cells. Materials Today. 2006;9(12):26-33 
14. Howard D, Buttery LD, Shakesheff KM, et al. Tissue engineering: strategies, stem cells and scaffolds. J Anat. 2018;13(1):66-72.

15. Mousavi SH, Abroun S, Soleimani M, et al. Expansion of human cord blood hematopoietic stem/ progenitor cells in three- dimensional Nanoscaffold coated with Fibronectin. Int J Hematol Oncol Stem Cell Res. 2015;9(2):72-79.

16. Mohamed A, Xing MM. Nanomaterials and nanotechnology for skin tissue engineering. Int J Burn Trauma. 2012;2(1):29-41.

17. Sahoo R, Jacob PJS, Sahoo S. Biomedical applications of green biopolymer guar gum. J Pharm Biomed Sci. 2013;35(35):1783-1787.

18. Minekus M, Jelier M, Xiao JZ, et al. Effect of partially hydrolyzed guar gum (PHGG) on the bioaccessibility of fat and cholesterol. Biosci Biotechnol Biochem. 2005;69(5):932-938.

19. Krishnaiah YS, Satyanarayana S, Rama Prasad YV, et al. Gamma scintigraphic studies on guar gum matrix tablets for colonic drug delivery in healthy human volunteers. J Control Release. 1998;5(2-3):245-252.

20. Roy I, Sardar M, Gupta NM. Cross-linked alginate-guar gum beads as fluidized bed affinity media for purification of jacalin. Biochem Eng J. 2005;3(3):193-198.

21. Pettolino AF, Hoogenraad NJ, Ferguson C, et al. A $(1 \rightarrow 4)$-beta-mannanspecific monoclonal antibody and its use in the immunocytochemical location of galactomannans. Planta. 2001;214(2):235-242.

22. Mafi R, Pelton R, Cui Y, et al. Weak gelation of hydrophobic guar by albumin in simulated human tear solutions. Biomacromolecules. 2014;15(12):4637-4642.

23. Shand FH, Langenbach SY, Keenan CR, et al. In vitro and in vivo evidence for anti-inflammatory properties of 2-methoxyestradiol. J Pharmacol Exp Ther. 2011;336(3):962-972.

24. Bognar E, Sarszegi Z, Szabo A, et al. Antioxidant and anti-inflammatory effects in raw 264.7 macrophages of malvidin, a major red wine polyphenol. PLoS One. 2013;8(6):e65355.
25. Wu D, Yotnda P. Induction and testing of hypoxia in cell culture. J Vis Exp. 2011;(54):2899.

26. Okail MSA. Cobalt chloride, a chemical inducer of hypoxia- inducible factor-1 $\alpha$ in U251 human glioblastoma cell line. Journal of Saudi Chemical Society. 2010;14(2):197-201.

27. Tendler DS, Bao C, Wang T, et al. Intersection of interferon and hypoxia signal transduction pathways in nitric oxide-induced tumor apoptosis. Cancer Res. 2001;61(9):3682-3688.

28. Cortizo AM, Ruderman G, Mazzni FN, et al. Novel Vanadium Loaded Ordered Collagen Scaffold Promotes Osteochondral Differentiation of Bone Marrow Progenitor Cells. International Journal of Biomaterials. 2016(2016):1-11.

29. Kuhbier JW, Allmeling C, Reimers K, et al. Interactions between Spider Silk and Cells - NIH/3T3 Fibroblasts Seeded on Miniature Weaving Frames. Plos One. 2010;5(8):e12032.

30. Hsieh WC, Liau JJ, Li YJ. Characterization and Cell Culture of a Grafted Chitosan Scaffold for Tissue Engineering. International Journal of Polymer Science. 2015(2015):1-7.

31. Izadpanah R, Trygg C, Patel B, et al. Biologic properties of mesenchymal stem cells derived from bone marrow and adipose tissue. $J$ Cell Biochem. 2006;9(5):1285-1297.

32. Eltzschig HK, Carmeliet P. Hypoxia and inflammation. N Engl J Med 2011;364(7):656-665.

33. Bartels K, Grenz A, Eltzschig HK. Hypoxia and inflammation are two sides of the same coin. Proc Natl Acad Sci USA. 2013;110(46):18351-18352.

34. Yu J, Yin S, Zhang W, et al. Hypoxia preconditioned bone marrow mesenchymal stem cells promote liver regeneration in a rat massive hepatectomy model. Stem Cell Res Ther. 2013;4(4):83.

35. Shi H, Guo Y, Liu Y, et al. The in vitro effect of lipopolysaccharide on proliferation, inflammatory factors and antioxidant enzyme activity in bovine mammary epithelial cells. Animal Nutrition. 2016;2(2):99-104. 